\title{
BANKNOTE RECOGNITION SYSTEM FOR VISUALLY IMPAIRED PEOPLE
}

\author{
Alaa Abdulfattah*, Abdurrahman A. Nasr, and A. Al-Marakeby \\ Systems and Computers Engineering Department, Faculty of Engineering, Al-Azhar \\ University,Nasr City, Cairo, Egypt. \\ *Corresponding Author E-mail:
}

\begin{abstract}
:
Paper currency recognition is one of the most important needs for visually impaired people to deal with different life circumstances. In this paper a layered framework is developed in which an Egyptian banknote recognition system is given. The developed system can recognize banknote in all geometric transformation (scaling, rotation, translation, shearing) and all lightning effects. The system is validated by using dataset which contains 1200 images. The underlying image dataset has been divided into five main categories (clear, rotated, noisy, folded and old images ).The system is tested and the recognition accuracy is $93 \%$ in all categories. The first layer in the framework deals with watermark security. The second layer of the framework, the segmentation has been applied, so that its value, special textures and special drawings are extracted. In the third layer of the framework, the local banknote features have been utilized. the fourth layer is a classification layer where different techniques are applied. Moreover, the developed system recognizes multiple banknotes. The implemented framework paves the way for various mobile applications are used effectively and commercially to assist visually impaired people.
\end{abstract}

\section{KEYWORDS: Banknote Recognition, Speeded up Robust Feature, Feature Detection and Description, eature Matching, Watermark Security, and Classification.}

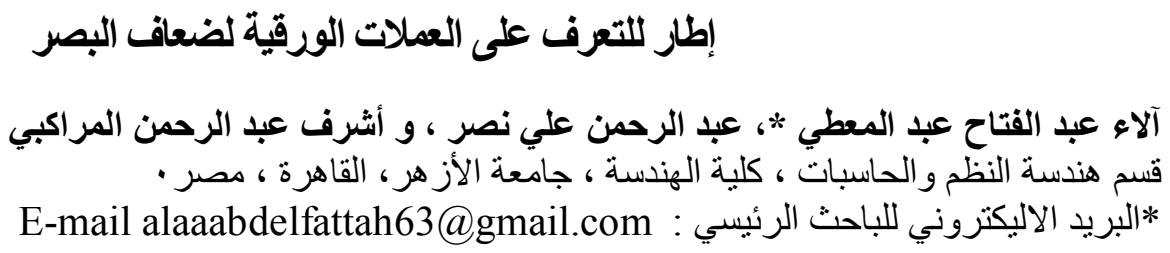
يعد التعرف المص: على العملات الورقية أحد أهم احتياجات الأشخاص ضعاف البصر للتعامل مع ظروف الحياة المختلفة. في هذا

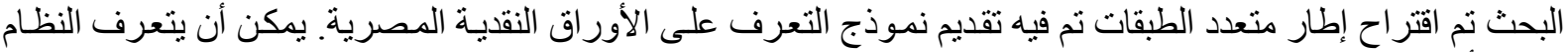

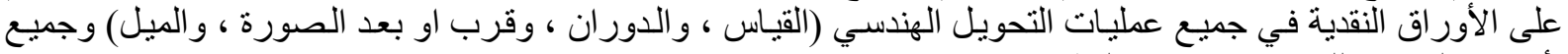

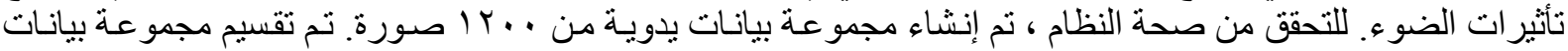

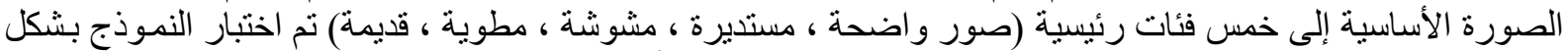

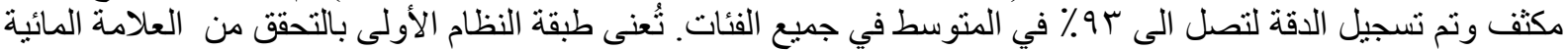

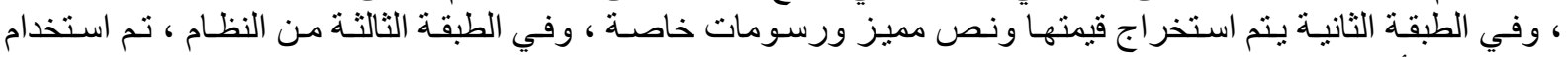
خصائص الأوراق النقدية المحلية . الطبقة الر ابعة هي طبقة تصنيف حيث يمكن تطبيق تقنيات مختلفة. علاوة على ذلك ، 


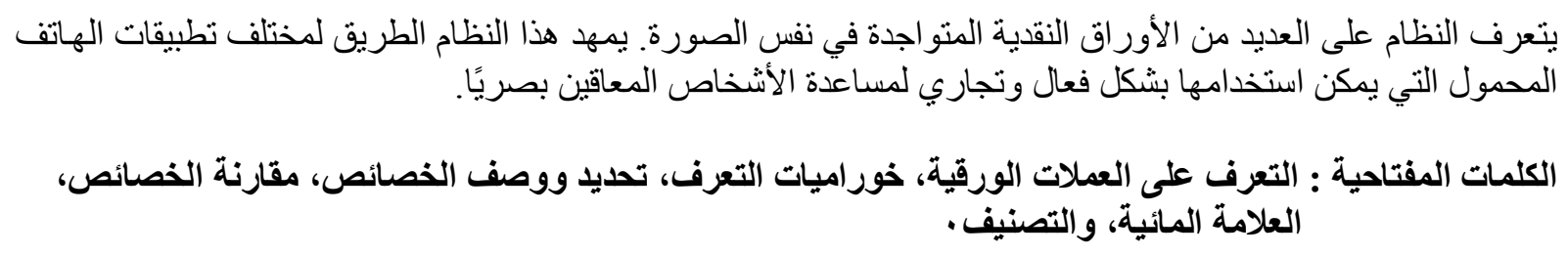

\section{INTRODUCTION}

Banknote recognition is one of the most important challenging problems that is faced by blind people. Generally ,banknote recognition systems should be able to:

- Identify the banknote watermark,

- $\quad$ Recognize the banknote in all geometric variations (scaling, rotation and translation) and in lighting effects.

- $\quad$ Classification of the banknote under consideration

Image pre-processing is an important step to improve the image quality .Bilateral filter and histogram equalization have been applied to improve the banknote image quality if image has been taken in low lightning condition. Bilateral filter has been applied as it preserves edges and blobs and they are important features in banknote image.

Accuracy and speed are important factors in banknote recognition systems(practically accuracy is more important than the speed. Recently there are many techniques for banknote recognition including Scale Invariant Feature Transform (SIFT) [1] and Speeded Up Robust Feature (SURF) [2] .The extraction process should be stable, efficient and distinctive in order to achieve robustness. As such, A 4-layer framework is implemented and validated using a private dataset of 1200 images. The dataset is divided into : clear, rotated, noisy, folded and old categories.

This paper is organized as follows .Section 2 provides a survey on related works while section 3 summarizes the SURF technique.Section 4 presents the proposed framework and section 5 discusses its implementation. section 6 is the conclusion of the paper

\section{RELATED WORK}

In recent years, several techniques have been developed to recognize banknote images to help visually impaired people. In 2003, Three Layer Perception Model and Radial Basis Function [3] has been applied to recognize Euro banknotes which was effective for image classification. The corresponding accuracy was reported to approach $85 \%$. Also in 2003, local Principal Component Analysis (PCA) [4] has been applied to recognize US Dollars. A learning vector quantization (LVQ) network is used as classifier .The Accuracy was reported to be $100 \%$.

In 2008, Edge Detection and Artificial Neural Network [5] have been applied to recognize Srilankan banknotes. In that work, Canny algorithm was used for edge detection as it has good ability to localize edges. In addition , a neural network is used for banknote image classification. The Accuracy was 85\%.In 2010, Negatively Correlated Neural Network Ensemble [6] has been applied to recognize Bangladeshi currency (TAKA) . Negative correlation learning has been used to provide the required training so that this method could recognize the banknote image even though the banknote image was noisy. Also In 2010, Block Local binary pattern algorithm [7] has been applied to recognize Chinese Currency(Yuan).Such Algorithm has been used for feature extraction from banknote images. In this method, the banknote recognition takes place in two phases. The first phase is creating a model by extracting important features from image. The second phase is classification phase by template matching .The accuracy was reported to be $100 \%$.

In 2011, Fast Adaboost Weak Classifier Training Algorithm [8]has been applied to recognize Chinese Currency(Yaun). This algorithm sort the eigen values from large to small, then traverse the sorted array once to find suitable threshold and bias. In this method the accuracy reached 97\%.In 2012, a Side Invariant Technique [9] has been applied to recognize Indian paper currency. the accuracy was reported to approach 99.5\%.Moreover, In 2012, Discrete Wavelet Transform (DWT) [10] has been applied to recognize Iranian and United Arab Emirates currency notes. Also, in 2012, an algorithm in which Weighted Euclidean Distance was combined with Neural Network [11] has been applied to recognize Bahrain Paper Currency. The accuracy of that algorithm was $96.4 \%$. 
In 2017, image processing techniques [12] have been applied to recognize Iranian banknote using a system that consists of two steps. In the first step, the banknote image is read as an RGB color model. In the second step, the RGB model is transformed to black and white models. Then by applying some image processing techniques on the banknote, its value of money is determined.

While this research was up and running, Hasanuzzaman etal [13] published their paper in which SURF is exploited to detect the salient features of US Dollars where the SURF implementation has reached $100 \%$ accuracy. In addition, in 2019 traditional and deep learning approaches has been relied upon to recognize automatically the Malaysian currency . in that work, the accuracy also reached $100 \%$

\section{3-SURF-Technique}

In this section SURF algorithm is discussed in explanatory steps based on the view point that SIFT algorithm [1] is the predecessor of SURF .Practically, SIFT is robust and reliable ,however, it is slow. To overcome the slowness of SIFT, SURF uses square-shaped filter as approximation to Gaussian smoothing . i.e. approximating the Laplacian of Gaussian filter by difference of Gaussian . Filtering the image with square is actually fast if the integral image, In, is used. Intuitively, the definition of integral image could be denoted in terms of summed area table which is a data structure for efficiently generating the sum of values in a rectangular subset of a grid. In the imaging counterpart it is known as integral image. Thus, the required sum $\mathrm{S}(\mathrm{x}, \mathrm{y})$ is given by :

$$
S(x, y)=\sum_{i=0}^{x} \sum_{j=0}^{y} \operatorname{In}(i, j)
$$

In equation 1 in the integral image, In, is used as a data structure to provide $S(x, y)$ of the original image .Thus squared shaped filters are used here as an approximation of Gaussian smoothing in SIFT accordingly as the integral image is used $\mathrm{S}(\mathrm{x}, \mathrm{y})$ of the original image is evaluated rapidly and effectively so that SURF can be pointed out as follows.

\section{1-Feature detection}

SURF uses a blob (Binary large object) detector based on the Hessian matrix [14]to find points of interest. The determinant of that Hessian matrix is used as a measure of local change around the point. The points to be considered are chosen where this determinant is maximum. SURF also uses the determinant of the Hessian for selecting the scale.A box filter of size $9 * 9$ is an approximation of a Gaussian with, Standard deviation , $\sigma=1.2$ and represents the lowest level (highest spatial resolution ) for blob response maps.

\section{2-Scale space representation and points of interest location}

The scale space is divided into a number of octaves, where an octave refers to a series of response maps of covering a doubling of scale. In SURF, the lowest level of the scale space is obtained from the output of $9 \times 9$ filters.

Hence, unlike previous methods, scale spaces in SURF are implemented by applying box filters of different sizes. Accordingly, the scale space is analyzed by up-scaling the filter size rather than iteratively reducing the image size. The output of the $9 \times 9$ filter is considered as the initial scale layer at scale $\mathrm{s}=1.2$ (corresponding to Gaussian derivatives with $\sigma=1.2$ ). The following layers are obtained by filtering the image with gradually bigger masks, taking into account the discrete nature of integral images and the specific filter structure. This results in filters of size $9 \times 9,15 \times 15,21 \times 21,27 \times 27, \ldots$ etc . The maxima of the determinant of the Hessian matrix are then interpolated in scale and image space with the method proposed by Brown, et al[13]. Scale space interpolation is essential important in this case, as the difference in scale between the first layers of every octave is relatively large.

\section{3-Providing descriptors}

The goal of a descriptor is to provide a unique and robust description of an image feature , e.g., by describing the intensity distribution of the pixels within the neighborhood of the point 
of interest. Most descriptors are thus computed in a local manner, hence a description is obtained for every point of interest identified previously.

The dimensionality of the descriptor has direct impact on both its computational complexity and point-matching robustness(accuracy) . A short descriptor may be more robust against appearance variations, but may not offer sufficient discrimination and thus give too many false positives.

\section{4-Oriantation assignment}

In order to achieve rotational invariance, the orientation of the point of interest needs to be found. The Haar wavelet [15] responses in both $\mathrm{x}$ - and $\mathrm{y}$-directions within a circular neighborhood of radius $\mathrm{R}$ around the point of interest are computed. The obtained responses are weighted by a Gaussian function centered at the point of interest, then plotted as points in a two-dimensional space, with the horizontal response in the abscissa and the vertical response in the ordinate. The dominant orientation is estimated by calculating the sum of all responses within a sliding orientation window of size $\pi / 3$. The horizontal and vertical responses within the window are summed. The two summed responses then yield a local orientation vector. The longest such vector overall defines the orientation of the point of interest. The size of the sliding window is a parameter that has to be chosen carefully to achieve a desired balance between robustness and angular resolution.

\section{5- Region Description Using The Sum Of Haar Wavelet Responses}

To describe the region around the point, a square region is extracted, centered on the interest point and oriented along the orientation as selected above. The interest region is split into smaller $4 \times 4$ square sub-regions, and for each one, the Haar wavelet responses are extracted at $5 \times 5$ regularly spaced sample points. The responses are weighted with a Gaussian (to offer more robustness for deformations, noise and translation). 6-Matching of descriptors obtained from different images By comparing the descriptors obtained from different images, matching pairs can be found. The pseudo code and Python program of the SURF algorithm can be found in [16].

\section{PROPOSED FRAMEWORK}

The proposed model consists of three layers, as shown in figure

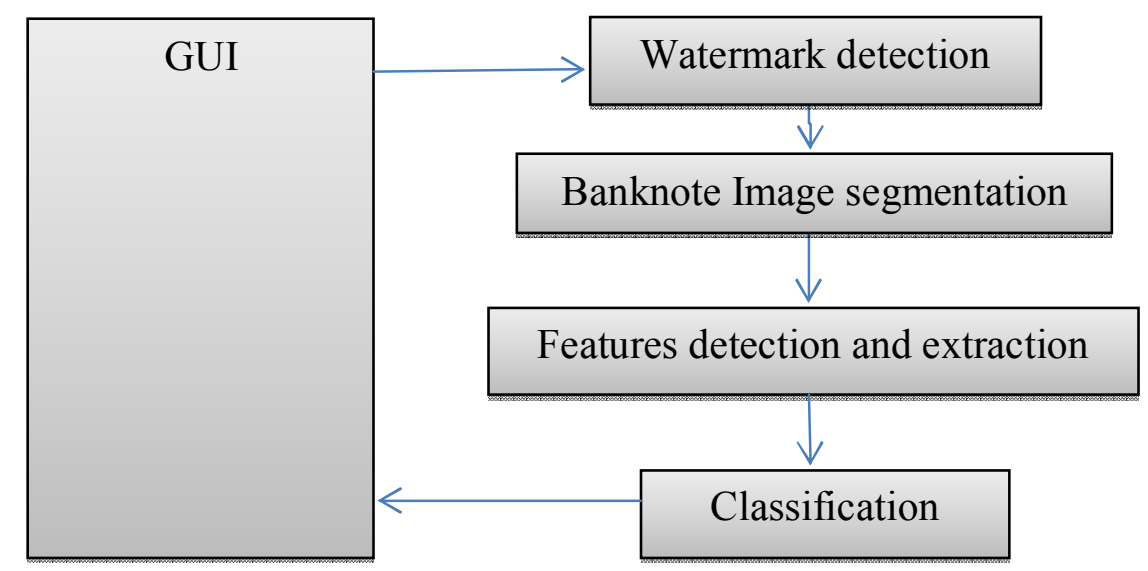

Fig 1:Banknote Recognition framework

a Graphical user interface is used to receive the user input and to demonstrate the system output. The first layer focuses on watermark extraction so that the user can decide early whether the underlying banknote is true or false. The second layer is a SURF based Egyptian Banknote Recognizer for Blind people(SEBRB) such layer comprises several computational sub layers. The third layer exploits machine learning to perform classification. 


\section{4-1 Watermark detection}

Unfortunately, the use of ultraviolet light, infrared and magnetic field to detect counterfeit currency are very cost. The use of digital image processing techniques are the simple and effective to detect the presence of watermark in banknote image.

A watermarked image $\mathbf{J}$ is achieved by imposing a watermark $\mathrm{W}$ over an unwatermarked image I with a blend factor, Yielding the following equation:

$$
J(p)=\alpha(p) W(p)+(1-\alpha(p)) I(p)
$$

Where $\mathrm{p}(\mathrm{x}, \mathrm{y})$ is the pixel location. For a set of $\mathrm{K}$ images,

$$
J_{k}=\alpha W+(1-\alpha) I_{k}, \quad k=1,2 \ldots K
$$

Although a lot of unknown quantities ( ) exist, we can make use of the structural properties of the image to determine its location and estimate its structure. The coherency of and W over all the images can be exploited to solve the above problem with good accuracy. The steps to determine these values are:

- $\quad$ Prepare initial watermark estimation and detection

- Calculate the median of the watermarked image gradients, independently in the $\mathrm{x}$ and $\mathrm{y}$ directions, at every pixel location $\mathrm{p}$.

$$
\nabla W_{m}(p)=\operatorname{median}_{k}\left(\nabla J_{k}(p)\right)
$$

- $\quad$ Crop Wm to remove boundary regions by computing its magnitude and taking the bounding box of the edge map. The initial estimated watermark is estimated using Poisson reconstruction

- Watermark detection: Obtain an edge map (using Canny edge detector) and compute its Euclidean distance transform, which is then correlated with to get the chamfer distance from each pixel to the closest edge. Lastly, the watermark position is taken to be the pixel with minimum distance in the map. The computer program of that watermark detection algorithm has not been implemented here since its implementation details are available in [19]

\section{4-2 Banknote image segmentation}

This step is applied to improve target banknote image quality if image captured in low lightning condition. Consequently, image masks has been introduced to mark the points of interest with white color the, to extract the region of interest from a banknote image the mask is ORed with Reference image.by using such technique (SEBRB) can process both noisy and occluded image.

\section{4-3 Features detection and extraction}

This layer is carried out using SURF based algorithm for Egyptian banknote recognition (SEBRB).The main steps of SEBRB are presented in figure 2.Initially, the images dataset has been divided into five categories ( clear, rotated, noisy, folded and old ), then the dataset is introduced to the model in order to determine the accuracy of each category. In the first step ,ideal images for each banknote (from both sides) have been taken as shown in figure 3 

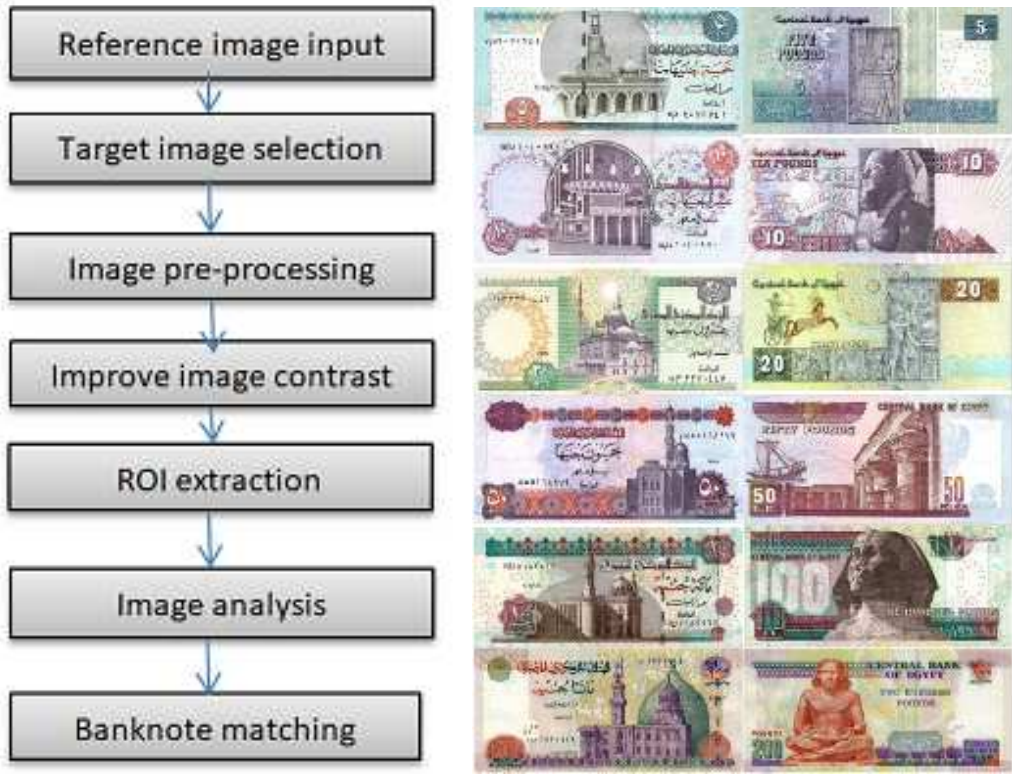

Fig. 2 SEBRB Flowchart Fig .3 The two sides of reference images for Egyptian banknotes

In order to start recognizing the target currency the camera of a smartphone is used to capture the required image. To speed up the input processing for each category, the proposed framework can recognize all images in specific directory and save results in text file. Consequently, target image is converted from colored image to gray image by making use of the following equation.

$$
\mathbf{J}=(\mathbf{R}+\mathbf{G}+\mathbf{B}) / \mathbf{3}
$$

To remove noise from target banknote image and improve quality of banknote recognition, bilateral filter is applied because it preserves the edges and blobs as important features in banknote images. The contrast of the target image is improved using a Contrast Limited Adaptive Histogram Equalization (CLAHE) .Figure 4 shows an Egyptian banknote before (Figure 4 a) and after (Figure $4 \mathrm{~b}$ ) contrast .

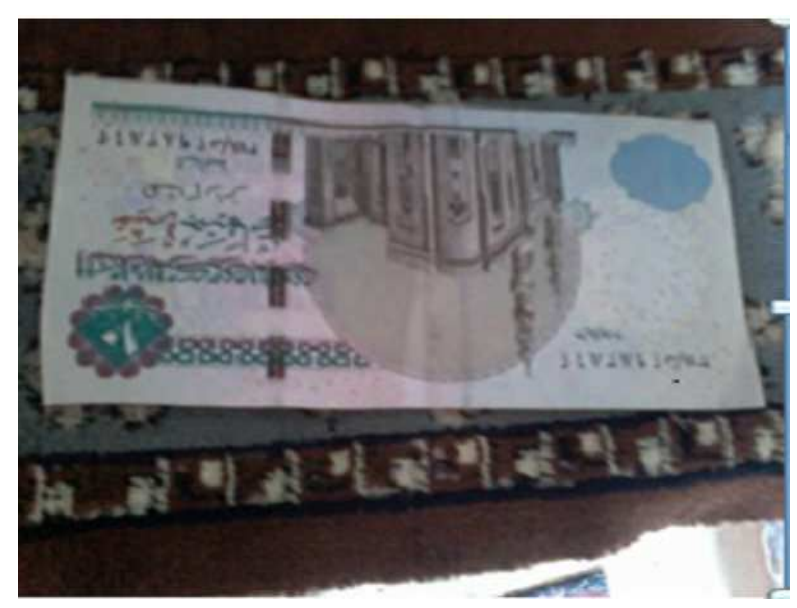

a)before contrast improvement

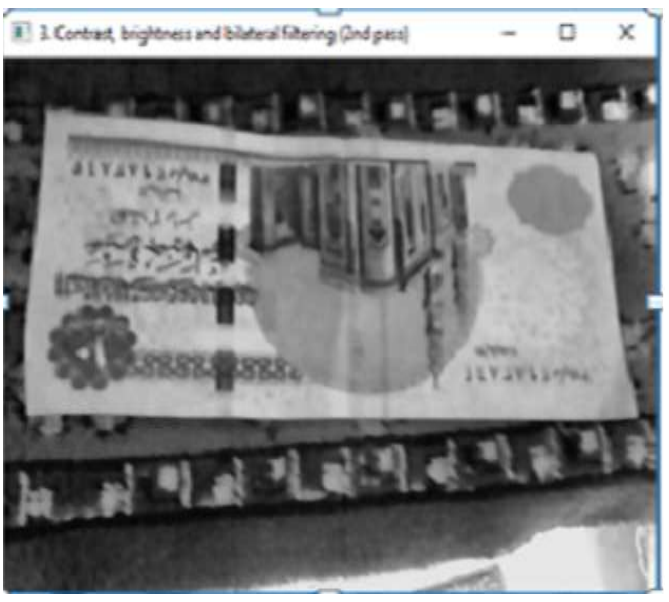

b)after contrast improvement

Fig 4. Contrast progress states,(a)before and (b)after improvement

Now, Speeded Up Robust Features (SURF) can be applied on the extracted Region of Interest, ROI, to achieve features detection and description with the advantage of being invariant for all geometric transformation such as Scaling, Rotation and Translation. Lastly, 
the banknote matching process is taken place where The matching between the target image and all reference images can be performed using the nearest neighbor approach.

\section{4-4 Classification}

The last layer of the framework is the classification layer where a machine learning approach either with traditional or deep learning is used .Feature extraction of the RGB values from banknote image of different regions were relied upon to support the classification algorithms such as k-Nearest Neighbors (KNN), Decision Tree (DT), Support Vector Machine (SVM) and Naive Bayesian Classifier (NBC) for recognizing each class of banknote.

These algorithms are available as Artificial intelligence programs in Weka[17].Also Banknote image with different orientation can be fed to a deep learning AlexNet [18].In that work of [18] Both kNN and DT achieved 99.7\% accuracy but both SVM and NBC perform better by achieving $100 \%$ accuracy. Also it has been found that deep learning provides reasonable results if only and only if the underlying network had previously been trained with data of similar orientation.

\section{5-Implementation}

The details of the implementation of the proposed framework including (SEBRB) is pointed out as follows.

\section{5-1 Setup}

The experimental setup is based on hardware that consists of Core i5,6 GB RAM. On that hardware python 2.7, OpenCV and sklearn libraries are established. On the basis of such environment a client-server prototype has been built. The client side is responsible for executing input/output using a smartphone android while the feature extraction and recognition processes are accomplished by the system server.

\section{5-2 Performance and results}

The performance of SEBRB is reported in table 1(a,b) .In these tables currencies with $5,10,20,50,100$ and $200 \mathrm{EGP}$ are examined after categorizing them to clear, rotated, Figure 5 i. Figure 6 shows an occluded image for 50 EGP banknote while Figure indicates that currency after rotation., fold and old .Both tables for front and back banknote images confirm the observation of accuracy decreasing of occluded, folded and old currencies either due to front or back banknote image.

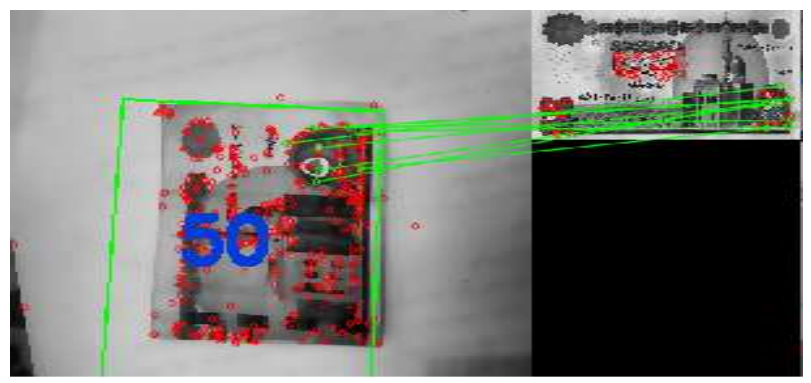

Fig 5: Sample of folded image

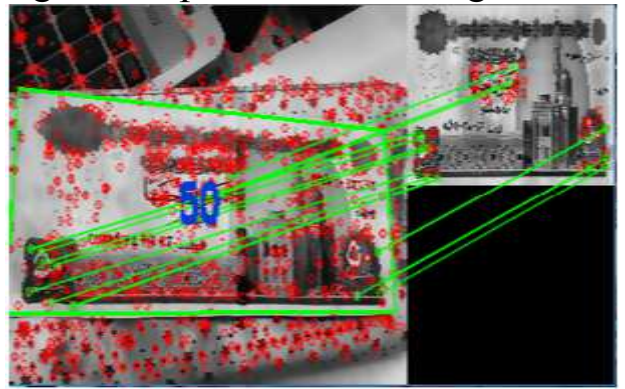

Fig 6: Samples of occluded images

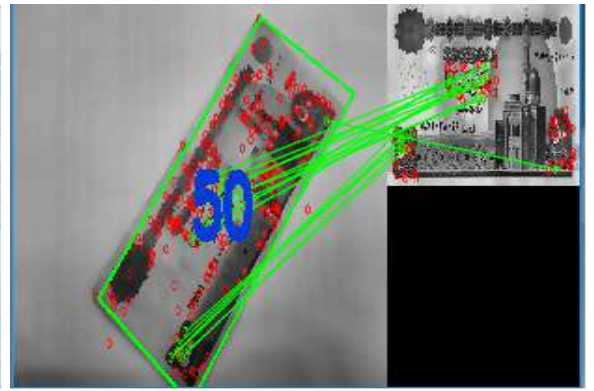

Fig 7: Sample of rotated image 
Table 1 : Accuracy of Egyptian banknote,(a)front images and (b)back images

\begin{tabular}{|l|l|l|l|l|l|l|}
\hline condition & 5 & 10 & 20 & 50 & 100 & 200 \\
& EGP & EGP & EGP & EGP & EGP & EGP \\
\hline Clear & $100 \%$ & $90 \%$ & $100 \%$ & $100 \%$ & $100 \%$ & $100 \%$ \\
\hline rotated & $95 \%$ & $100 \%$ & $90 \%$ & $100 \%$ & $100 \%$ & $95 \%$ \\
\hline occluded & $80 \%$ & $95 \%$ & $90 \%$ & $90 \%$ & $85 \%$ & $90 \%$ \\
\hline folded & $100 \%$ & $85 \%$ & $85 \%$ & $100 \%$ & $100 \%$ & $95 \%$ \\
\hline old & $100 \%$ & $85 \%$ & $90 \%$ & $95 \%$ & $80 \%$ & $100 \%$ \\
\hline
\end{tabular}

\begin{tabular}{|l|l|l|l|l|l|l|}
\hline condition & $\begin{array}{l}5 \\
\text { EGP }\end{array}$ & $\begin{array}{l}10 \\
\text { EGP }\end{array}$ & $\begin{array}{l}20 \\
\text { EGP }\end{array}$ & $\begin{array}{l}50 \\
\text { EGP }\end{array}$ & $\begin{array}{l}100 \\
\text { EGP }\end{array}$ & $\begin{array}{l}200 \\
\text { EGP }\end{array}$ \\
\hline Clear & $95 \%$ & $100 \%$ & $100 \%$ & $100 \%$ & $100 \%$ & $100 \%$ \\
\hline rotated & $90 \%$ & $100 \%$ & $95 \%$ & $100 \%$ & $100 \%$ & $90 \%$ \\
\hline occluded & $90 \%$ & $85 \%$ & $80 \%$ & $95 \%$ & $80 \%$ & $90 \%$ \\
\hline folded & $100 \%$ & $85 \%$ & $90 \%$ & $100 \%$ & $95 \%$ & $100 \%$ \\
\hline old & $90 \%$ & $80 \%$ & $100 \%$ & $95 \%$ & $80 \%$ & $95 \%$ \\
\hline
\end{tabular}

a) Accuracy of front Banknote images

b) Accuracy of Front Banknote images

\section{5-3 Discussion}

The performance of SEBRB, Table 1(a,b) has been focused on the Egyptian banknote recognition, therefore, it does not make sense to compare it with other similar works that are interested in the recognition of other national currencies due to using different datasets and operational parameters. nevertheless, the work in [13], Table 2, depends on SURF to execute banknote recognition by the same way that has been employed here. These works, as well as other similar trials give an obvious proof of concept of using SURF for banknote recognition.

Table 2 : Accuracy of US Dollars [13]

\begin{tabular}{|c|c|c|c|}
\hline $\begin{array}{c}\text { Ground } \\
\text { Truth }\end{array}$ & $\begin{array}{c}\text { No. of } \\
\text { images }\end{array}$ & $\begin{array}{c}\text { False } \\
\text { Recognition } \\
\text { Rate }\end{array}$ & $\begin{array}{c}\text { True } \\
\text { Recognition } \\
\text { Rate }\end{array}$ \\
\hline$\$ 1$ & 20 & $\mathbf{0}$ & $\mathbf{1 0 0 \%}$ \\
\hline$\$ 2$ & 20 & $\mathbf{0}$ & $\mathbf{1 0 0 \%}$ \\
\hline$\$ 5$ & 20 & $\mathbf{0}$ & $\mathbf{1 0 0 \%}$ \\
\hline$\$ 10$ & 20 & $\mathbf{0}$ & $\mathbf{1 0 0 \%}$ \\
\hline$\$ 20$ & 20 & $\mathbf{0}$ & $\mathbf{1 0 0 \%}$ \\
\hline$\$ 50$ & 20 & $\mathbf{0}$ & $\mathbf{1 0 0 \%}$ \\
\hline$\$ 100$ & 20 & $\mathbf{0}$ & $\mathbf{1 0 0 \%}$ \\
\hline
\end{tabular}

In [18] two different approaches are examined, one depends on SURF while the other exploits deep learning. The investigations given in [18]confirm the result that the SURF approach can be relied upon with the advantages of avoiding laborious learning, sophisticated software , expensive hardware and sensitivity to image orientation (rotation ) relative to deep learningbased approach.

\section{CONCLUSION}

This paper presents a 4-layer framework represented by Watermark security, Banknote Image Segmentation ,Feature detection/ description and banknote classification on the basis of that framework SEBRB is carried out as a client-server smartphone application in which the mobile phone is responsible for the input-output processing while the SURF algorithm is executed on the system server. The accuracy is reported to reach up $93 \%$ in all categories.

The proposed-SURF based recognizer has the advantages that: - it is robust and effective it emphasizes the criticality-it compensates for various geometric and lighting parameters. In addition local matching is applied to handle occluded, noisy and old Egyptian banknote . in handling old, folded and occluded banknote. It affords valuable, reliable and friendly tool for visually impaired people to easily recognize Egyptian banknote.

\section{REFERENCES}

1. Low, D. G.,( 2004). "Distinctive image features from scale-invariant keypoints," Int. J. Comput. Vis., pp. 91-110 [Online]. Available https://www.cs.ubc.ca/ lowe papers / /ijcv04.pdf.

2. Leonardis,A., Bischof,H., Pinz,A., Bay,H., Tuytelaars,T., and Van Gool, L., July 2006 "Computer Vision - ECCV 2006 SURF: Speeded Up Robust Features," Comput. Vis. - ECCV 2006, vol. 3951, no., pp. 404-417-417, 2006, doi: 10.1007/11744023. 
3. Aoba, M., Kikuchi, T., and Takefuji, Y.,( May 2003). "Euro banknote recognition system using a three-layered perceptron and RBF networks," IPSJ Trans. Math. Model. Appl, vol. 44, no., pp. 99-109.

4. Ahmadi, A., Omatu, S., and Kosaka, T.,( 2003) "A Reliable Method for Recognition of Paper Currency by Approach to Local PCA," Proc. Int. Jt. Conf. Neural Networks, vol. 2, pp. 1258-1262, , doi: 10.1109/ijenn..1223874.

5. Gunaratna D. A. K. S., Kodikara N. D., and Premaratne H. L.,( 2008) "ANN Based Currency Recognition System using Compressed Gray Scale and Application for Sri Lankan Currency Notes - SLCRec," Eng. Technol., vol. 2, no. 9, pp. 235-240,.

6. Debnath, K. K., Ahdikary, J. K., and Shahjahan, M.,( 2009). "A currency recognition system using negatively correlated neural network ensemble," ICCIT 2009 - Proc. 2009 12th Int. Conf. Comput. Inf. Technol., no. ICCIT, pp. 367-372, doi: 10.1109/ICCIT.2009.5407265.

7. Guo J., Zhao Y., and Cai A.,(2010). "A reliable method for paper currency recognition based on LBP," Proc. - 2010 2nd IEEE Int. Conf. Netw. Infrastruct. Digit. Content, IC-NIDC 2010, pp. 359-363, doi: 10.1109/ICNIDC.2010.5657978.

8. Dqj H. W., Gu L., and Du L., (2011)“Adaboost training algorithm,” pp. 4772-4775,.

9. C. B. V Vijaya and Vijaya P. A., "A Robust Side Invariant Technique of Indian Paper Currency Recognition," Int. J. Eng. Res. Technol., vol. 1, no. 3, pp. 1-7, 2012, [Online]. Available: www.ijert.org.

10. Rajaei A., Dallalzadeh E., and Imran M.( 2012), "Feature extraction of currency notes: An approach based on wavelet transform," Proc. - 2012 2nd Int. Conf. Adv. Comput. Commun. Technol. ACCT 2012, pp. 255-258, , doi: 10.1109/ACCT.2012.53.

11. Althafiri E., Sarfraz M., and Alfarras M.( 2012), "Bahraini paper currency recognition," J. Adv. Comput. Sci. Technol. Res., vol. 2, no. 2, pp. 104-115.

12. Panah N. and Masoumi H.,( 2017) "Banknotes detected using Image Processing Techniques," Int. J. Comput. Sci. Mob. Comput., vol. 6, no. 5, pp. 34-44,.

13. Hasanuzzaman F. M., Yang X., and Tian Y.,( 2012). "Robust and effective component-based banknote recognition for the blind," IEEE Trans. Syst. Man Cybern. Part C Appl. Rev., vol. 42, no. 6, pp. 1021-1030, , doi: 10.1109/TSMCC.2011.2178120.

14. Swapnali B. B. and Vijay K. S.,( 2014) "Feature Extraction Using Surf Algorithm for Object Recognition,” Int. J. Tech. Res. Appl., vol. 2, no. 4, pp. 2320-8163,.

15. Porwik P. and Lisowska A., (2004)"The new graphic description of the Haar wavelet transform," Lect. Notes Comput. Sci. (including Subser. Lect. Notes Artif. Intell. Lect. Notes Bioinformatics), vol. 3039, pp. 1-8, , doi: 10.1007/978-3-540-25944-2_1.

16. Mordvintsev A. and Abid K.,( 2017). "OpenCV-Python Tutorials Documentation," OpenCV Python Doc., pp. 1-269, [Online]. Available: https://media.readthedocs.org/pdf/opencv-python-tutroals/latest/opencv-pythontutroals.pdf.

17. Aksenova S. S., (2006).“WEKA Explorer Tutorial,” Calif. State Univ. Sacramento, vol. 11, no. 1, pp. 1-37, doi: 10.1007/s10115-007-0114-2.

18. Sufri N. A. J., Rahmad N. A., Ghazali N. F., Shahar N., and As'Ari M. A.,( August,2019). "Vision Based System for Banknote Recognition Using Different Machine Learning and Deep Learning Approach," ICSGRC 2019 - 2019 IEEE 10th Control Syst. Grad. Res. Colloquium, Proceeding, no. pp. 5-8 , doi: 10.1109/ICSGRC.2019.8837068.

19. https://github.com/rohitrango/automatic-watermark-detection accessed 30/6/2020 